\title{
Enhancement of Noisy Images with Sliding Discrete Cosine Transform
}

\author{
Vitaly Kober ${ }^{1}$ and Erika Margarita Ramos Michel $^{2}$ \\ ${ }^{1}$ Department of Computer Science, CICESE, Ensenada, B.C. , Mexico \\ vkober@cicese.mx \\ ${ }^{2}$ University of Colima, Colima, Mexico \\ ramemeucol.mx
}

\begin{abstract}
Enhancement of noisy images using a sliding discrete cosine transform (DCT) is proposed. A minimum mean-square error estimator in the domain of a sliding DCT for noise removal is derived. This estimator is based on a fast inverse sliding DCT transform. Local contrast enhancement is performed by nonlinear modification of denoised local DCT coefficients. To provide image processing in real time, a fast recursive algorithm for computing the sliding DCT is utilized. The algorithm is based on a recursive relationship between three subsequent local DCT spectra. Computer simulation results using a real image are provided and discussed.
\end{abstract}

\section{Introduction}

Many different image enhancement techniques have been introduced to improve the visual appearance of images [1-7]. These techniques may be broadly divided in two classes. The first class is based on decomposing an image onto high- and lowfrequency signals, manipulating them separately and then combining them. Examples of such methods are homomorphic filtering [1] and unsharp masking [2]. The second class consists of various histogram modification techniques [3]. The classical unsharp masking is one of the most commonly used methods for image enhancement because it works well in many real applications. In this method a fraction of the highfrequency signal of an image is added to the original image itself to form a locally enhanced image. Drawbacks of the unsharp masking are as follows. A linear highpass filter makes the system very sensitive to noise. This results in undesirable noise enhancement in flat and high-contrast areas of even slightly noisy images. The operation also uses a constant scaling factor that may lead to overshoot artifacts in high-contract areas of the image. Various methods have been proposed to improve the performance of the unsharp masking [4-6]. The use of quadratic filters instead of a linear highpass filter enhances details and edges in accordance with a human perceptual criterion. These filters can be described as local mean weighted highpass filters. Weighting the highpass filter output by the local mean value leads to enhancement in dark areas less than that of in bright areas. This coincides with Weber's law, which states that the just noticeable brightness difference is proportional to average background brightness. Consequently the perceived noise is reduced 
comparing with that of the unsharp masking output. These methods have all proven to be effective in local enhancing of images. However, the methods discussed above use a moving window with fixed size and shape (usually $3 \times 3$ pixels). This may not be equally effective in enhancing various structures in an image, which can vary widely in size and shape. Examples such structures are fine edges and middle-sized details. Moreover, these structures can be degraded due to different kinds of noise. Obviously that additive noise could be better suppressed using a large moving window. Thus there is a need to design a technique, which preserves and enhances different-sized structures in the original image, while eliminating noise. Methods using a spatially adaptive filter mask (neighborhood), whose size and shape are adjustable to local details to be processed, are appropriate for this purpose. The price for the adaptivity to the local signal is a high computational complexity. Recently, some algorithms with adaptive filter masks for image enhancement were proposed [7]. A highpass filtering is considered as an enhanced difference between the central pixel of a moving window and a smoothed version of the original image. The smoothing is performed with rank-order filters over various adaptive neighborhoods defined around the central pixel of a moving window.

In this paper, we carry out enhancement of noisy images using a sliding discrete cosine transform (DCT) coefficients. The sliding DCT is based on the concept of short-time signal processing [8]. The short-time orthogonal transform of a signal $x_{k}$ is defined as

$$
X_{s}^{k}=\sum_{n=-\infty}^{\infty} x_{k+n} w_{n} \psi(n, s),
$$

where $w_{n}$ is a window sequence, $\psi(n, s)$ represents the basis functions of an orthogonal transform. In this paper we use one-dimensional notation for simplicity. Equation (1) can be interpreted as the orthogonal transform of $x_{k+n}$ as viewed through the window $w_{n} . X_{s}^{k}$ displays the orthogonal transform characteristics of the signal around time $k$. Note that while increased window length and resolution are typically beneficial in the spectral analysis of stationary data, for time-varying data it is preferable to keep the window length sufficiently short so that the signal is approximately stationary over the window duration. It is of interest to note that there is a link between wavelet and sliding short-time transforms. Wavelet transforms [9] are signal sub-band decompositions by filters with frequency responses formed from the Fourier transform of a mother function on a given scale. On the other hand, short-time signal transformation is a signal sub-band formed by a filter with frequency response equal to the Fourier transform of the windowed function of used orthogonal transform. The main distinction between these two sub-band decompositions is that the short-time transform decomposes signal into sub-bands of the same width uniformly arranged in the base, while sub-bands of the wavelet are arranged in logarithm scale and have width that is doubled with the increase of its frequency. Now we assume that the window has finite length around $n=0$, and it is unity for all $n \in\left[-N_{l}, N_{2}\right]$. Here $N_{l}$ and $N_{2}$ are integer values. This leads to signal processing in a sliding window [10]. In other words, local filters in the domain of an orthogonal transform at each position of a moving window modify the orthogonal transform coefficients of a signal to obtain only an estimate of the pixel $x_{k}$ of the window. The choice of orthogonal transform for 
sliding signal processing depends on many factors. The DCT is one the most appropriate transform with respect to the accuracy of power spectrum estimation from the observed data that is required for local filtering, the filter design, and computational complexity of the filter implementation. Linear filtering in the domain of DCT followed by inverse transforming is superior to that of the discrete Fourier transform (DFT) because a DCT can be considered as the DFT of a signal evenly extended outside its edges. This consequently attenuates boundary effects caused by circular convolution that are typical for linear filtering in the domain of DFT.

The proposed algorithm at each position of a moving window consists of two steps: first, noise suppression is performed in the domain of a sliding DCT, and then local contrast enhancement is carried out with nonlinear modification of denoised local DCT coefficients. The size of a moving window determines the maximum size of details to be enhanced. The presentation is organized as follows. In Section 2, we review recursive algorithms for computing the sliding forward and inverse DCTs. In Section 3, a local adaptive filter minimizing the minimum mean-square error defined in the domain of the sliding DCT is derived. In section 4 , we test the filter performance to enhance a real aerial image. Section 5 summarizes our conclusions.

\section{Fast Forward and Inverse Algorithms of Sliding DCT}

The discrete cosine transform is widely used in many signal processing applications such as adaptive filtering, video signal processing, feature extraction, and data compression. This is because the DCT performs close to the optimum Karhunen-Loeve transform for the first-order Markov stationary data, when the correlation coefficient is near 0.9 [11]. Recently, fast forward and inverse algorithms for fast computing of DCTs were proposed [12]. The sliding cosine transform (SCT) is defined as

$$
X_{s}^{k}=\sum_{n=-N_{1}}^{N_{2}} x_{k+n} \cos \left(\pi \frac{\left(n+N_{1}+1 / 2\right) s}{N}\right),
$$

where $N=N_{1}+N_{2}+1,\left\{X_{s}^{k} ; s=0,1, \ldots, N-1\right\}$ are the transform coefficients around time $k$. The coefficients of the DCT can be obtained as $\left\{C_{0}^{k}=X_{0}^{k} / \sqrt{2} ; C_{s}^{k}=X_{s}^{k}, s=1, \ldots\right.$, $N-1\}$. The SCT on the base of a recursive relationship between three subsequent local DCT spectra [12] is given by

$$
X_{s}^{k+1}=2 X_{s}^{k} \cos \left(\frac{\pi s}{N}\right)-X_{s}^{k-1}+\cos \left(\frac{\pi s}{2 N}\right)\left(x_{k-N_{1}-1}-x_{k-N_{1}}+(-1)^{s}\left(x_{k+N_{2}+1}-x_{k+N_{2}}\right)\right) \text {. (3) }
$$

We see that the computation of the DCT at the window position $k+1$ involves values of the input sequence $x_{k}$ as well as the DCT coefficients computed in two previous positions of the moving window.

Table 1. Number of arithmetical operations for computing of sliding DCT

\begin{tabular}{|c|c|c|}
\hline & Number of additions & Number of multiplications \\
\hline Fast DCTs [13] & $3 \mathrm{MN} / 2-\mathrm{N}+1$ & $\mathrm{MN} / 2+1$ \\
\hline Recursive algorithm & $2 \mathrm{~N}+5$ & $2 \mathrm{~N}-1$ \\
\hline
\end{tabular}


Tables 1 provides a comparison of the computational complexity of the recursive algorithm with fast DCT algorithms. The length of a moving window for the recursive algorithm is an arbitrary integer value determined by characteristics of a signal to be processed. In contrast, fast DCT algorithms require the length to be of a power of 2, $\mathrm{N}=2^{\mathrm{M}}$. If $x_{k}$ is the central pixel of the window, that is, $N_{l}=N_{2}$ and $N=2 N_{1}+1$, then the inverse transform is written as

$$
x_{k}=\frac{1}{N}\left(2 \sum_{s=1}^{N_{1}}(-1)^{s} X_{2 s}^{k}+X_{0}^{k}\right) .
$$

We note that in the computation only the spectral coefficients with even indices are involved. The computation requires one multiplication and $N_{l}+1$ additions.

\section{Signal Denoising in the Domain of Sliding DCT}

First we define a local criterion of the performance of filters for image and signal processing and then derive optimal local adaptive filters with respect to the criterion. One the most used criterion in signal processing is the minimum mean-square error (MMSE). Since the processing is carried out in a moving window, then for each position of a moving window an estimate of the central element of the window is computed. Suppose that the signal to be processed is approximately stationary within the window. The signal may be distorted by sensor's noise.

Let us consider a generalized linear filtering of a fragment of input onedimensional signal (for instance for a fixed position of the moving window). Let $\boldsymbol{a}=\left[a_{k}\right]$ be undistorted real signal, $\boldsymbol{x}=\left[x_{k}\right]$ be observed signal, $k=1, \ldots, N, N$ be the size of the fragment, $\boldsymbol{U}$ be the matrix of the discrete cosine transform, $E\{$.$\} be the expected$ value, superscript ${ }^{\mathrm{T}}$ denotes the transpose. Let $\overline{\boldsymbol{a}}=\boldsymbol{H} \boldsymbol{x}$ be a linear estimate of the undistorted signal, which minimizes the MMSE averaged over the window

$$
M M S E=E\left\{(\boldsymbol{a}-\overline{\boldsymbol{a}})^{T}(\boldsymbol{a}-\overline{\boldsymbol{a}})\right\} / N .
$$

The optimal filter for this problem is the Wiener filter [11]:

$$
\boldsymbol{H}=E\left\{\boldsymbol{a} \boldsymbol{x}^{T}\right\}\left[E\left\{\boldsymbol{x} \boldsymbol{x}^{T}\right\}\right]^{-1} .
$$

Let us consider the known model of signal:

$$
x_{k}=\sum_{n} w_{k, n} a_{n}+v_{k}
$$

where $\boldsymbol{W}=\left[w_{k, n}\right]$ is a distortion matrix, $\boldsymbol{v}=\left[v_{k}\right]$ is additive noise with zero mean, $k, n=1, \ldots N, N$ is the size of fragment. The equation can be rewritten as

$$
\boldsymbol{x}=\boldsymbol{W a}+\boldsymbol{v},
$$

and the optimal filter is given by

$$
\boldsymbol{H}=\boldsymbol{K}_{a a} \boldsymbol{W}^{T}\left[\boldsymbol{W} \boldsymbol{K}_{a a} \boldsymbol{W}^{T}+\boldsymbol{K}_{v v}\right]^{-1},
$$

where $\boldsymbol{K}_{a a}=E\left\{\boldsymbol{a} \boldsymbol{a}^{T}\right\}, \boldsymbol{K}_{v v}=E\left\{\boldsymbol{v} \mathbf{v}^{T}\right\}, E\left\{\boldsymbol{a} \mathbf{v}^{T}\right\}=0$ are the covariance matrices. It is assumed that an input signal and noise are uncorrelated. 
The obtained optimal filter is based on an assumption that an input signal within the window is stationary. The result of filtering is the restored window signal. This corresponds to signal processing in non-overlapping fragments. Now suppose that the signal is processed in a moving window in the domain of the sliding DCT. For each position of the window an estimate of the central pixel should be computed. Using the equation for inverse sliding DCT presented in the previous section, the point-wise MSE for reconstruction of the central element of the window can be written as follows:

$$
\operatorname{PMSE}(k)=E\left\{[a(k)-\bar{a}(k)]^{2}\right\}=E\left\{\left[\sum_{l=1}^{N} \alpha(l)(A(l)-\bar{A}(l))\right]^{2}\right\},
$$

where $\overline{\boldsymbol{A}}=[\bar{A}(l)=H(l) X(l)]$ is a vector of signal estimate in the domain of the DCT, $\boldsymbol{H}_{U}=[H(l)]$ is a diagonal matrix of the scalar filter, $\boldsymbol{\alpha}=[\alpha(l)]$ is a diagonal matrix of the coefficients of inverse sliding cosine transform (4). Minimizing (10), we obtain

$$
\boldsymbol{H}_{U}=\left[\boldsymbol{P}_{x x}\right]^{-1} \boldsymbol{P}_{a x} \boldsymbol{I}_{\alpha}
$$

where $\boldsymbol{P}_{a x}=[E\{A(l) X(k)\}], \boldsymbol{P}_{x \boldsymbol{x}}=[E\{X(l) X(k)\}], \boldsymbol{I}_{\boldsymbol{\alpha}}$ is the identity matrix of the dimension of $\boldsymbol{\alpha}$. Note that matrix of coefficients $\boldsymbol{\alpha}=[\alpha(l)]$ for the inverse sliding transform (4) is singular. The inverse sliding cosine transform (4) possesses the dimension of the matrix twice less than the size of the window signal. Therefore, the computational complexity of the scalar filters in (11) and signal processing can be significantly reduced comparing to the complexity for the filter in (6). For the model of signal distortion in (8) the filter matrix is given as

$$
\boldsymbol{H}_{U}=\left[\boldsymbol{U}\left(\boldsymbol{W} \boldsymbol{K}_{a a} \boldsymbol{W}^{T}+\boldsymbol{K}_{v v}\right) \boldsymbol{U}^{T}\right]^{-1} \boldsymbol{U} \boldsymbol{K}_{a a} \boldsymbol{W}^{T} \boldsymbol{U}^{T} \boldsymbol{I}_{\alpha} .
$$

If a signal has a high correlation coefficient and a smoothed version of the signal is corrupted by additive, weakly-correlated noise, then the matrix $\boldsymbol{U}\left(\boldsymbol{W} \boldsymbol{K}_{a a} \boldsymbol{W}^{T}+\boldsymbol{K}_{v v}\right) \boldsymbol{U}^{T}$ in (12) is close to diagonal. Figure 1 shows the covariance matrix of a smoothed, noisy, one-dimensional signal having the correlation coefficient of 0.95 as well as the discrete cosine transform of the covariance matrix. The linear convolution between a signal $\boldsymbol{x}$ and the matrix $\boldsymbol{K}_{a a} \boldsymbol{W}^{T}$ in the domain of the sliding DCT can be well approximated by a diagonal matrix $\operatorname{Diag}\left(\boldsymbol{U} \boldsymbol{K}_{a a} \boldsymbol{W}^{T} \boldsymbol{U}^{T} \boldsymbol{I}_{\alpha}\right) \boldsymbol{X}$. Therefore, the matrix of the scalar filter in (12) is close to diagonal, and the filter can be written as

$$
H(l) \approx \frac{P_{1}(l)}{P_{2}(l)+P_{v v}(l)},
$$

where $P_{1}(l), P_{2}(l), P_{n n}(l)$ are diagonal elements of the following matrices $\boldsymbol{U} \boldsymbol{K}_{a a} \boldsymbol{W}^{T} \boldsymbol{U}^{T} \boldsymbol{I}_{\alpha}, \boldsymbol{U} \boldsymbol{W} \boldsymbol{K}_{a a} \boldsymbol{W}^{T} \boldsymbol{U}^{T}, \boldsymbol{U} \boldsymbol{K}_{v v} \boldsymbol{U}^{T}, \quad l=1, \ldots N_{l}, N_{l}$ is the dimension of the matrix $\boldsymbol{I}_{\alpha}$. 


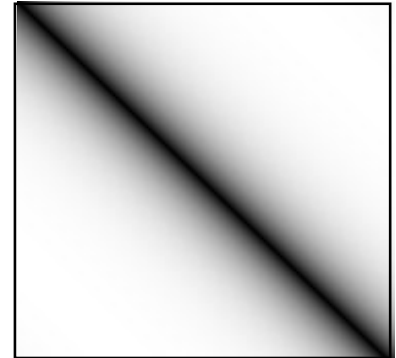

(a)

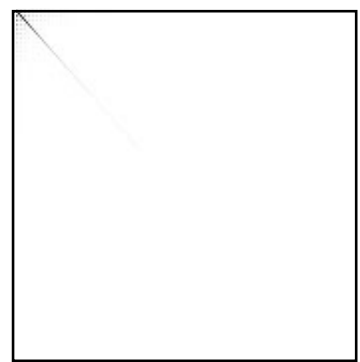

(b)

Fig. 1. (a) Covariance matrix of a noisy signal, (b) DCT of the covariance matrix

For the design of local adaptive filters in the domain of a sliding DCT the covariance matrices and power spectra of fragments of a signal are required. Since they are often unknown, in practice, these matrices can be estimated from observed signals [14].

\section{Image Enhancement in the Domain of Sliding DCT}

The objective of this section is to develop a technique for noise suppression and enhancement local details on the base of a sliding DCT, and to test the algorithm performance in a real image. We design local adaptive filters to enhance noisy image. Assume that a clean image signal $\left\{a_{k}\right\}$ is degraded by zero-mean additive noise $\left\{v_{k}\right\}$ :

$$
x_{k}=a_{k}+v_{k},
$$

where $\left\{x_{k}\right\}$ is a noisy observed image. This is a particular case of (8) when $\boldsymbol{W}=\boldsymbol{I}$.

Let $\left\{X_{l}^{k}, A_{l}^{k}, V_{l}^{k}, \bar{A}_{l}^{k} ; l=1, \ldots, N\right\}$ be the DCT transform coefficients around time $k$ of noisy signal, clean signal, noise, and filtered signal, respectively. Here $N=2 N_{l}+1$ is the length of the DCT. Note that $N_{1}$ is an arbitrary integer value, which is determined by the minimal size of details to be preserved after filtering.

Various criteria can be exploited to design a filter for noise removal. We use the criterion of the PMSE around time $k$ which is defined in the domain of sliding DCT. An estimate of the reconstructed image signal with respect to the PMSE can be written as

$$
\bar{A}^{k}(l)=\left\{\begin{array}{ll}
\left(1-\frac{P_{v V}^{k}(l)}{P_{x x}^{k}(l)}\right) X^{k}(l), P_{x x}^{k}(l)>P_{v V}^{k}(l) \\
0, & \text { otherwise }
\end{array} .\right.
$$

where $P_{x x}^{k}(l) \approx\left\langle\left|X^{k}(l)\right|^{2}\right\rangle, P_{v v}^{k}(l) \approx\left\langle\left|V^{k}(l)\right|^{2}\right\rangle$ are estimates of the power spectra of the observed signal and noise in the domain of the sliding DCT, $\langle$.$\rangle is the average$ operation over signal fragments, $l=1, \ldots, N_{l}$. The obtained filter can be considered as a spectral subtraction method in the domain of sliding DCT. In general, spectral 
subtraction methods, while reducing the wide-band noise, introduce a new narrowband noise due to the presence of remaining spectral peaks. To attenuate the remaining noise, one can suggest over subtraction of the power spectrum of noise by introducing a nonzero power spectrum bias.

Image enhancement may be regarded as an extension of image restoration methods. However, in contrast to image restoration, image enhancement often requires intentional distorting image signal such as increasing local contrast. In order to carry out image enhancement, we use a modification of local spectra in the domain of the sliding DCT. Finally, the processed image signal can be written as follows:

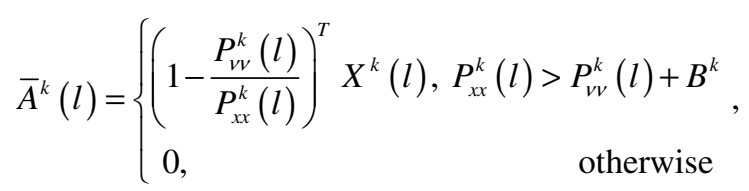

where $B^{k}$ is a signal-dependent bias value, $T$ is a parameter. When $0 \leq T \leq 1$, the modification of the local spectra redistributes of the energy of spectral coefficients in favor of low energy coefficients. Note that in real images the spectral coefficients of high frequency often possess low energy. The filtered image can be obtained with the use of the inverse DCT transform. Note that in the processing only the spectral coefficients with even indices are involved.

A real low contrast and noisy aerial image is shown in Fig. 2 (a). The size of image is $256 \times 256$, each pixel has 256 levels of quantization. The image is corrupted by zeromean additive Gaussian noise. The objective of our computer experiment is to enhance meddle-sized details (about 15x15) in the noisy image.

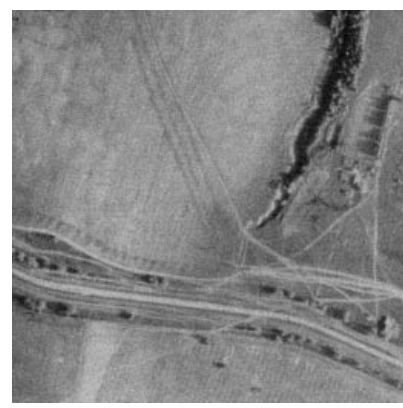

(a)

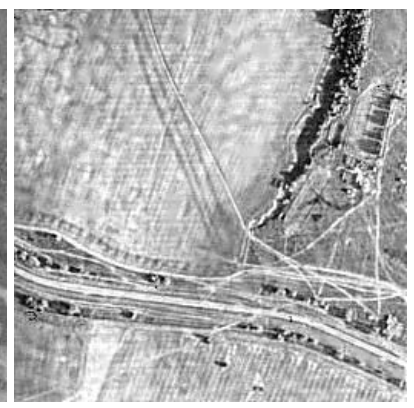

(b)

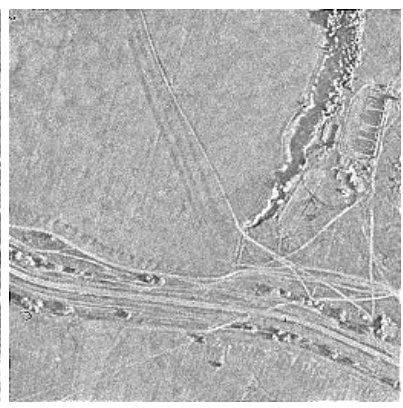

(c)

Fig. 2. (a) Noisy aerial image, (b) denoised and enhanced image in the domain of sliding DCT, (c) denoised and enhanced image in the domain of global DCT

It is difficult to define a criterion to accurately quantify the performance of algorithms. Ramponi [4] proposed to calculate the local variance of the original and processed images over the pixels of $3 \times 3$ moving window. However, this method is appropriate for edge or fine detail enhancement in noise-free images. Since the objective of local detail enhancement is an improvement of visual appearance of 
details, we use a subjective visual criterion. In our tests the window length of $15 \times 15$ pixels and $T=0.25$ is used. Since there exists difference in spectral distributions of the image signal and wide-band noise, the power spectrum of noise can be easily measured from the experimental covariance matrix [14]. The result of noise filtering and local contrast enhancing on the observed image with the proposed filter is shown in Fig. 2 (b). Fig 2 (c) shows the result of noise suppression and enhancement with the same algorithm designed in the domain of DCT of the entire image. We see that the proposed algorithm is capable to perform simultaneous noise suppression and local contrast enhancement, whereas the result of the global filtering is not satisfactory.

\section{Conclusions}

In this paper, we have presented a new technique for enhancing images degraded by additive noise. The technique utilizes the sliding DCT. In order to provide image processing in real time, a fast recursive algorithm for computing the sliding DCT was utilized. Extensive testing has shown that additive noise can be significantly reduced as well as local contrast enhanced by proper choice of algorithm parameters.

\section{References}

1. Oppenheim A.V., Shafer R.W. and Stockham Jr. T.G., Nonlinear filtering of multiplied and convolved signals, Proc. IEEE, Vol. 56, No. 8, (1968) 1264-1291.

2. Schreiber W.F., Wirephoto quality improvement by unsharp masking, Pattern recognition, Vol. 2, No.4, (1970) 117-121.

3. Hummel R.A., Image enhancement by histogram transformation, Comp. Graph. Image process., Vol. 6, (1977) 184-195.

4. Ramponi G., Strobel N., Mitra S.K., and Yu T., Nonlinear unsharp masking methods for image contrast enhancement, J. Electron. Imag., Vol. 5, (1996) 353-366.

5. Ramponi G., A cubic unsharp masking technique for contrast enhancement, Signal Processing, Vol. 67, (1998) 211-222.

6. Polesel A., Ramponi G., Mathews V.J., Image enhancement via unsharp masking, IEEE Trans. Image Process., Vol. 9, No. 3, (2000) 505-510.

7. Kober V., Mozerov M., Alvarez-Borrego J., Ovseyevich I.A., Unsharp masking using rank-order filters with spatially adaptive neighborhoods, Pattern Recognition and Image Analysis, Vol. 12, No.1, (2002) 46-56.

8. Oppenheim A.V., Shafer R.W., Discrete-time signal processing, Prentice Hall, Englewood Cliffs, NJ (1989).

9. Mallat S., A wavelet tour of signal processing, Academic Press, NY (1999).

10. Vitkus R.Y., and Yaroslavsky L.P., Recursive algorithms for local adaptive linear filtration, in: Mathematical Research., Academy Verlag, Berlin, (1987) 34-39.

11. Jain A.K. Fundamentals of digital image processing. Prentice Hall, NY (1989).

12. Kober V., Fast algorithms for the computation of sliding discrete sinusoidal transforms, IEEE Trans. on Signal Process., Vol. 52, No 6, (2004) 1704-1710.

13. Hou H.S. A fast recursive algorithm for computing the discrete cosine transform, IEEE Trans. Acoust. Speech Signal Process., Vol. 35, No. 10, (1987) 1455-1461.

14. Yaroslavsky L., Eden M. Fundamentals of digital optics. Birkhäuse, Boston (1996). 